\title{
O processamento das informações sociais em crianças e adolescentes agressivos
}

\author{
Silvio José Lemos Vasconcellos \\ Faculdades de Taquara (RS) \\ Patrícia Picon \\ Pontifícia Universidade Católica do Rio Grande do Sul \\ Laura Pithan Prochnow \\ Universidade Luterana do Brasil - Santa Maria, RS \\ Gabriel José Chittó Gauer \\ Pontifícia Universidade Católica do Rio Grande do Sul
}

\begin{abstract}
Resumo
Este artigo tem por objetivo revisar a literatura internacional publicada no período de 1980 até 2004 sobre o Modelo de Processamento das Informações Sociais, utilizando as palavras-chave cognição social e processamento de informações sociais (PsycINFO). Os autores analisam a convergência desses estudos quanto ao processamento das informações sociais por crianças e adolescentes agressivos. De acordo com o modelo, a resposta comportamental de uma criança ou adolescente para um estímulo social problemático ocorre em função de diferentes etapas de processamento: codificação das pistas sociais, interpretação das pistas sociais, busca de respostas, avaliação das respostas e atuação. Após uma breve introdução histórica sobre o modelo e seus principais postulados, são descritos alguns estudos empíricos sobre o tema. Uma relação entre o processamento de informações sociais e a manifestação de comportamentos agressivos foi confirmada nesta revisão, mas verificou-se uma baixa incidência de artigos contemplando amostras clínicas.
\end{abstract}

Palavras-chave: comportamento agressivo; processamento de informações; cognição social

\begin{abstract}
The processing of social information by aggressive children and adolescents. This paper aimed to review the international literature on Social Information Processing Model published from 1980 to 2004 using the keywords social cognition and information-processing social (PsycINFO). The authors analyze the convergence of these studies in regard to information processing by aggressive children and adolescents. According to the model, a child and adolescent's behavioral response to a problematic social stimulus is a function of different steps of processing: encoding of social cues, interpretation of social cues, response search, response evaluation, and enactment. After a short historical introduction about the model and its main postulates, empirical studies were described. The relation between social information-processing and aggressive behavior was confirmed in this revision but a low incidence of articles based on clinical samples was noted.

Keywords: aggressive behavior; information-processing; social cognition
\end{abstract}

I números estudos têm evidenciado a existência de algumas particularidades relacionadas à forma como crianças e adolescentes agressivos processam as informações sociais. Em termos gerais, essas pesquisas estão voltadas para uma tentativa de melhor especificar o modo como alguns aspectos da cognição social contribuem para a manifestação de comportamentos agressivos em seus diferentes níveis. São contempladas, portanto, questões relativas à codificação dos dados sociais, à interpretação dos mesmos, bem como o próprio processo de gerar respostas diante de uma situação de interação social e tomar decisões sobre a adoção das mesmas.

Neste artigo, são revisadas, de forma sistemática, pesquisas realizadas a partir da década de 80 que contemplam diferentes questões relacionadas ao processamento das informações sociais em crianças e adolescentes agressivos com base no modelo proposto por Dodge (1986) e na sua respectiva reformulação (Crick \& Dodge, 1994). A revisão contemplou o período de 1980 até 2004, utilizando os descritores processamento de informações sociais e cognição social 
indexados no PsycINFO. Por intermédio deste trabalho, procurou-se investigar a concordância desses estudos quanto à possibilidade de evidenciar particularidades nas diferentes etapas de processamento das informações sociais verificadas em crianças e adolescentes agressivos.

Os artigos que serão discutidos ao longo desta revisão remetem a um modelo que, embora não seja o único na esfera da cognição social, vem sendo significativamente discutido e investigado por uma série de pesquisas nas últimas décadas. Em termos gerais, são analisados trabalhos que precederam e contribuíram para a elaboração das diferentes versões do modelo em questão, bem como aqueles que sucederam e estiveram fundamentados nessas mesmas versões. Foram incluídos todos os estudos que realizaram qualquer tipo de análise experimental sobre as diferentes etapas do processamento das informações sociais, sendo que alguns estudos descritivos também são referidos ao longo do trabalho, porém, tão somente com o objetivo de melhor explicar o modelo em questão. Na seqüência, serão explicadas as versões do citado modelo.

\section{O modelo cognitivo de processamento de informações sociais em suas diferentes versões}

Com base numa série de dados empíricos coletados sobre o modo como crianças e adolescentes agressivos processam as informações sociais, Keneth Dodge elaborou o chamado modelo social de processamento de informação da competência em crianças (Dodge, 1986). Em termos gerais, o referido modelo descreve uma seqüência de operações cognitivas relativas à forma como os dados sociais são tratados. O autor procurou, desse modo, sintetizar o tipo de seqüência que perfaz o processamento das informações sociais, levando em conta a atuação de capacidades biologicamente determinadas, bem como o repertório de informações presentes e atuantes nesse processo. As etapas descritas nesse modelo são, respectivamente: a codificação dos dados sociais, a representação dos mesmos, a busca por respostas apropriadas, a decisão sobre tais respostas e o processo de atuação propriamente dito.

De acordo com esse modelo, certos déficits nos diferentes mecanismos responsáveis pelo processamento das informações sociais acabam por sustentar um padrão de comportamentos agressivos, na medida em que se verifica um prejuízo na própria habilidade de resolver situações de impasse que tendem a ocorrer no dia-a-dia. Segundo Flavell, Miller e Miller (1999), uma das principais características dessa abordagem refere-se ao fato de que o comportamento socialmente inadequado pode ter causas distintas que podem ir desde a codificação tendenciosa até uma falha na tradução de uma decisão em um comportamento. Trata-se, portanto, de um entendimento sobre o modo como o sujeito irá servir-se dos dados sociais a fim de gerar comportamentos específicos, sendo que o caráter seqüencial do processamento poderia ser descrito como uma das suas principais características (Crick \& Dodge, 1994).

Numa tentativa de ampliar esse modelo e aproximá-lo das assim chamadas modelagens conexionistas desenvolvidas no âmbito das ciências cognitivas, Crick e Dodge (1994) acabaram por desenvolver o modelo reformulado de processamento das informações sociais de ajustamento em crianças. Nesse novo modelo, são descritas seis etapas: codificação de dicas internas e externas, interpretação e representação mental destas dicas, clarificação ou seleção de um objetivo, construção ou acesso à resposta, decisão da resposta e realização do comportamento. Conforme salientam Kristensen, Lima, Ferlin, Flores e Hackmann (2003), o modelo apresenta um entendimento sobre o caráter cíclico da agressividade, uma vez que a criança acaba por receber um retorno sobre o seu comportamento na etapa seis. Nesses termos, a reformulação do modelo mostra-se capaz de melhor explicar o próprio ciclo da violência ressaltado por Dodge, Bates e Pettit (1990). Crick e Dodge (1994) salientam também que a reformulação proposta contempla, de forma mais apropriada, o papel das emoções no tocante ao processamento. Segundo os autores: "Em concordância com essa linha de raciocínio, é proposto, no modelo reformulado apresentado, que as emoções são parte integrante de cada passo no processamento das informações sociais" (Crick \& Dodge, 1994, p. 81). Cada etapa mostra-se, nesse sentido, sujeita a um processo de coativação com base em esquemas sociais pré-existentes, processo esse que se mostra em concordância com o próprio entendimento conexionista sobre a organização do conhecimento (Smolensky, 1990). Conforme destaca Pakaslahti (2000), o modelo em questão tornou-se um dos principais modelos teóricos para explicar os mecanismos subjacentes ao comportamento agressivo em crianças e adolescentes.

Na seqüência deste artigo, são revisados estudos que procuraram investigar o modo como crianças e adolescentes agressivos processam as informações sociais a partir das diferentes etapas mencionadas. Para tanto, buscou-se agrupar tais estudos de acordo com os aspectos avaliados da cognição social relativos, por sua vez, a uma ou mais etapas constituintes do modelo reformulado desenvolvido por Crick e Dodge (1994). Em alguns casos, os estudos são referenciados mais de uma vez, tendo em vista que, nesses casos, uma diversidade de etapas foi investigada. Destacaram-se ainda alguns estudos que se propuseram a investigar a confiabilidade do modelo de processamento das informações sociais mencionado.

\section{Avaliação das etapas de codificação e de interpretação dos dados sociais}

Dodge e Newman (1981) evidenciaram o fato de que crianças com tendências agressivas mostravam-se menos eficazes no tocante à codificação das informações sociais. Conforme esse estudo, as crianças não agressivas do grupo controle demonstraram, em termos quantitativos, uma habilidade maior para perceber e identificar as informações que se fazem presentes num contexto interpessoal. Investigações realizadas por Lochman e Dodge (1994) também evidenciaram o fato de que garotos agressivos demonstravam uma menor capacidade de lembrar dos dados relevantes envolvidos numa situação interpessoal. Estudos desenvolvidos por Shahinfar, 
Kupersmidt e Matza (2001), bem como por Slaby e Guerra (1988) evidenciaram problemas na etapa de codificação das dicas com adolescentes institucionalizados. Gouze (1987) destacou que adolescentes agressivos prestam mais atenção a situações em que há indícios de manifestação de agressividade do que seus pares. Dodge, Murphy e Buchsbaum (1984) mostraram que o ato de ser, por exemplo, atingido por trás com uma bola nas proximidades de um jogo tende a ser interpretado por crianças agressivas como sendo intencional. De um modo geral, uma maior tendência para atribuir hostilidade por parte de crianças e adolescente agressivos foi também verificada em uma série de estudos (Dodge \& Frame, 1982; Dodge \& Tolmin, 1987; Graham \& Hudley, 1994; Guerra \& Slaby, 1989; e Steinberg \& Dodge, 1982). Um estudo que procurou avaliar essa tendência em crianças agressivas a partir do modo como estas eram descritas pelas próprias mães também encontrou confirmações significativas (Dix \& Lochman, 1990). Hubbard, Dodge, Cillessen, Coie e Schwartz (2001), bem como Dodge e Coie (1987) evidenciaram, no entanto, que a atribuição de hostilidade mostra-se mais característica em crianças que manifestam uma agressão reativa do que naquelas que manifestam uma agressão pró-ativa. Uma pesquisa que procurou relacionar o estado afetivo induzido com determinadas tendências na etapa de interpretação permitiu ainda mostrar o quanto tal processo depende também do estado afetivo (Bryan, Sullivan \& Mathur, 1988). Por outro lado, o estudo desenvolvido por Bryan, Sullivan e Mathur (1988) não relacionou, de forma direta, a manifestação da agressividade com a própria tendência para manter certos estados afetivos.

Dentre os estudos que investigaram populações mais específicas, destacam-se os trabalhos de Dodge, Pettit, Bates e Valente (1995), pelo qual ficou demonstrado que vítimas de abuso tendiam a gerar equívocos de codificação e atribuição de hostilidade num grau maior do que seus pares. Diferenças desse tipo também foram encontradas em crianças com retardo mental quando comparadas a grupo controle (Gómez \& Hazeldine, 1996). Milich e Dodge (1984) demonstraram, por sua vez, que crianças hiperativas apresentavam uma maior tendência em atribuir hostilidade para situações de impasse do que seus pares. Quiggle, Garber, Panak e Dodge (1992) demonstraram que crianças depressivas tendiam a uma maior atribuição de hostilidade do que crianças normais.

\section{Avaliação das etapas de seleção de objetivos, construção e decisão sobre as respostas}

No que se refere ao número total de respostas cogitadas para determinadas situações de interação, um conjunto de trabalhos evidenciou que crianças e adolescentes agressivos demonstram uma maior limitação. (Lochman \& Dodge, 1994; Mize \& Cox, 1990; Pettit, Dodge \& Brown, 1988; Richard \& Dodge, 1982; Slaby \& Guerra, 1988). Shahinfar, Kupersmidt e Matza (2001) evidenciaram que adolescentes institucionalizados, em geral, tendem a esperar mais resultados positivos quanto à adoção de estratégias agressivas do que seus pares. Uma série de evidências empíricas quanto ao fato de que crianças e adolescentes agressivos demonstram ser mais favoráveis à adoção de estratégias agressivas também foi obtida por intermédio de diferentes pesquisas (Asarnow \& Callan, 1985; Guerra \& Slaby, 1989; Huesman \& Guerra, 1997; Liska, Richard, Chamlin, \& Baccaglini, 1984; Pakaslahti \& Keltikangas-Jarvinen, 1998; Slaby \& Guerra, 1988; Quiggle, Garber, Panak, \& Dodge, 1992). Richard e Dodge (1982) demonstraram ainda que crianças agressivas tendem a referenciar uma estratégia agressiva antes das demais estratégias cogitadas para uma situação hipotética de impasse. O trabalho de Pettit, Dodge e Brown (1988) demonstrou, por sua vez, que a capacidade de gerar e decidir sobre respostas para situações de impasse está significativamente relacionada às experiências anteriores da criança em seu ambiente familiar. Um dos estudos evidenciou também que crianças agressivas tendem a esperar menos resultados positivos ao cogitar a adoção de atitudes pró-sociais (Quiggle et al., 1992). Mayeux e Cillessen (2003) evidenciaram que a sofisticação das estratégias citadas está diretamente relacionada com a idade, bem como com o fato da criança ser aceita ou rejeitada diante dos seus pares.

Quanto aos estudos que procuraram avaliar essas etapas de processamento para populações específicas, destacase a pesquisa de Gómez e Hazeldine (1996), que também encontrou limitações na quantidade de respostas agressivas mencionadas por parte de crianças com retardo mental. Precariedades na capacidade de gerar respostas foram evidenciadas nos trabalhos de Milich e Dodge (1984) com crianças hiperativas. Num trabalho que investigou meninas com problemas de aprendizagem, foi possível identificar uma correlação entre dificuldade de aprendizagem e a capacidade para decidir sobre as respostas geradas em situações de impasse (Tur-Kaspa, 2004). A tendência para conceber a adoção de estratégias agressivas como fazendo parte das soluções mais eficazes para situações de impasse foi verificada em crianças vítimas de abuso nos estudos de Dodge e colaboradores (1995). Por outro lado, um estudo que avaliou homens agressivos com um nível moderado de retardo mental não atestou existir diferença na quantidade de respostas geradas, mas tão somente na freqüência com que as estratégias agressivas eram referenciadas (Fuchs \& Benson, 1995). Já nos estudos em que o status social dos avaliados foi levado em consideração, ficou demonstrado que crianças consideradas rejeitadas pelos seus pares tendem a possuir expectativas mais positivas quanto à adoção de estratégias agressivas (Feldman \& Dodge, 1987; Hart, Ladd, \& Burleson, 1990).

\section{Estudos que investigaram a confiabilidade do modelo}

Foi encontrado um estudo longitudinal que procurou relacionar as diferentes deficiências em etapas do processamento das informações sociais com a manifestação de agressividade ao longo do desenvolvimento das crianças avaliadas. Burks, Laird, Dodge, Pettit e Bates (1999) avaliaram uma amostra de meninos e meninas acompanhados desde o jardim de infância até a $8^{\underline{a}}$ série, sendo que, por intermé- 
dio desse acompanhamento, foi possível mostrar que deficiências no processamento das informações sociais contribuem para a manutenção dos comportamentos agressivos tanto em crianças de menor idade como em crianças mais velhas. Dodge, Laird, Lochman e Zelli (2002) procuraram, de outro modo, realizar um estudo confirmatório sobre a validade do modelo considerando as suas diferentes etapas e a consistência interna para as modalidades de processamento. Nesse trabalho, comprovou-se a multidimensionalidade do modelo, a sua consistência interna e a sua capacidade preditiva para padrões de comportamentos agressivos com base em inventários preenchidos por professores. Um estudo que procurou avaliar as diferentes etapas de processamento para situações específicas envolvendo interação com pares e figuras de autoridade, relacionando ainda essa performance com descrições da competência social para as crianças avaliadas, encontrou confirmações parciais para o modelo. Já um estudo que utilizou a Rosenzweig Picture-Frustration StudyChildren's Form (PFSCF) para avaliar a atribuição de hostilidade e, dessa forma, buscar correlações com comportamentos agressivos descritos por professores demonstrou a existência de correlações significativas entre essas descrições e os desempenhos verificados nas testagens (Graybill \& Heuvelman, 1993).

\section{Conclusão}

Em termos gerais o pressuposto de que particularidades específicas nas etapas de processamentos de informações sociais relacionam-se com a manifestação de comportamentos agressivos em crianças e adolescentes pode ser atestado por inúmeras pesquisas conforme essa revisão mostrou. Por intermédio do presente trabalho, pode-se também observar que houve uma produção ativa nas duas décadas estudadas, bem como no início da presente década, sendo que, nos anos oitenta, publicou-se um número maior de trabalhos com base no citado modelo, ainda que tal diferença não se mostre significativa.

Uma maior tendência para atribuir hostilidade, uma interpretação mais tendenciosa das situações de interação social e uma maior limitação quanto à capacidade de gerar e decidir sobre as possíveis respostas adotadas para situações de impasse por parte de crianças e adolescentes agressivos ficaram bem evidenciadas diante dos estudos destacados. Diferença na quantidade de respostas geradas não foram, entretanto, encontradas em uma amostra de homens adultos, com histórico de comportamentos agressivos e com retardo mental moderado (Fuchs \& Benson, 1995). Os estudos revisados atestam particularidades nas distintas etapas tanto para crianças como para adolescentes, embora um estudo tenha mostrado que a idade pode contribuir para a sofisticação das estratégias mencionadas (Mayeux \& Cillessen, 2003). Nesse sentido, demonstra ser pertinente uma melhor investigação da transição da adolescência para a idade adulta e as possíveis mudanças que se verificam em termos de processamento das informações sociais nos indivíduos considerados agressivos.
Estudos que possam melhor comparar diferenças sutis quanto às etapas de processamento em meninos e em meninas também podem mostrar-se oportunos, uma vez que esta revisão destacou o fato de ainda existir um número modesto de pesquisas com amostras de ambos os sexos.

Investigar até que ponto crianças e adolescentes considerados agressivos tendem a referenciar estratégias agressivas antes de qualquer outra estratégia parece ser também uma questão que merece uma avaliação complementar. Quanto à questão dos estudos com populações específicas, entende-se, por outro lado, que inúmeras pesquisas podem e devem ser realizadas. Os diferentes transtornos que acometem crianças e adolescentes e que, por sua vez, relacionam-se com a manifestação da agressividade podem ser avaliados e relacionados com o desempenho nas diferentes etapas de processamento das informações sociais.

De um modo geral, os estudos de confiabilidade revisados demonstram que o modelo elaborado por Dodge (1986) e reformulado por Crick e Dodge (1994) possui capacidade preditiva quanto à manifestação da agressividade na infância e na adolescência e pode, dessa forma, explicar certos mecanismos cognitivos que contribuem para um dos problemas que mais demandam atenção da sociedade na época em que vivemos.

\section{Referências}

Asarnow, J. R., \& Callan, J. W. (1985). Boys with peer adjustment problems: social cognitive processes. Journal of Consulting and Clinical Psychology, $53,80-87$.

Bryan, T., Sullivan-Burstein, K., \& Mathur, S. (1988). The influence of affect on social-information processing. Journal of Learning Disabilities, 31, 418-426.

Burks, V. S., Laird, R. D., Dodge, K. A., Pettit, G. S., \& Bates, J. E. (1999). Knowledge structures, social information processing, and children's aggressive behavior. Social Development, 8, 220-236.

Crick, N. R., \& Dodge, K. A. (1994). A review and reformulation of social information-processing mechanisms in children's social adjustment. Psychological Bulletin, 115, 74-101.

Dix, T., \& Lochman, J. E. (1990). Social cognition and negative reactions to children: a comparison of mothers of aggressive and nonaggressive boys. Journal of Social and Clinical Psychology, 9, 418-438.

Dodge, K. A. (1986). A social information processing model of social competence in children. In M. Perlmutter (Org.), Minnesota Symposium in Child Psychology. Cognitive perspectives in children's social and behavioral development (Vol. 18, pp. 77-125). Hillsdale, New Jersey: Erlbaum.

Dodge, K. A., Bates, J. E., \& Pettit, G. S. (1990). Mechanisms in the cycle of violence. Science, 250, 1678-1683.

Dodge, K. A., \& Coie, J. D. (1987). Social-information-processing factors in reactive and proactive aggression in children's peer groups. Journal of Personality and Social Psychology, 53, 1146-1158.

Dodge, K. A., \& Frame, C. L. (1982). Social cognitive biases and deficits in aggressive boys. Child Development, 53, 620-635

Dodge, K. A., Laird, R., Lochman, J. E., \& Zelli, A. (2002). Multidimensional latent-construct analysis of children's social information processing patterns: correlations with aggressive behavior problems. Psychological Assessment, 14, 60-73.

Dodge, K. A., Murphy, R. M., \& Buchsbaum, K. (1984). The assessment of intention-cue discrimination cues in children: implications for developmental psychopathology. Child Development, 55, 163-173. 
Dodge, K. A., \& Newman, J. P. (1981). Biased decision making processes in aggressive boys. Journal of Abnormal Psychology, 90, 375-379.

Dodge, K. A., Pettit, G. S., Bates, J. E., \& Valente, E. (1995). Social informationprocessing patterns partially mediate the effect of early physical abuse on later conduct problems. Journal of Abnormal Psychology, 104, 632-643.

Dodge, K. A., \& Tolmin, A. M. (1987). Utilization of self-schemas as a mechanism of interpretational bias in aggressive children. Social Cognition, 5, 280-300.

Feldman, E., \& Dodge, K. A. (1987). Social information processing and sociometric status: sex, age and situational effects. Journal of Abnormal Child Psychology, 15, 211-227.

Flavell, J. H., Miller, P. H., \& Miller, S. A. (1999). Desenvolvimento cognitivo ( $3^{\underline{a}}$ ed.). Porto Alegre: Artmed.

Fuchs, C., \& Benson, B. A. (1995). Social information processing by aggressive and nonaggressive men with mental retardation. American Journal of Mental Retardation, 100, 244-252.

Gómez, R., \& Hazeldine, P. (1996). Social information processing in mild mentally retarded children. Research in Developmental Disabilities, 17, 217-227.

Gouze, J. R. (1987). Attention and social problem-solving as correlates of aggression in preschool males. Journal of Abnormal Child Psychology, 15, 181-197.

Graybill, D., \& Heuvelman, L. R. (1993). Validity of children's picture-frustration study: a social cognitive perspective. Journal of Personality Assessment, 60, 379-389.

Graham, S., \& Hudley, C. (1994). Attributions of aggressive and nonagressive African-American male early adolescents: a study of construct accessibility. Development Psychology, 30, 365-373.

Guerra, N. G., \& Slaby, R. G. (1989). Evaluative factors in social problem-solving by aggressive boys. Journal of Abnormal Child Psychology, 17, 277-289.

Hart, C. H., Ladd, G. W., \& Burleson, B. (1990). Children's expectations of the outcomes of social strategies: relations with sociometric status and maternal disciplinary styles. Child Development, 61, 127-137.

Hubbard, J. A., Dodge, K. A., Cillessen, A. H., Coie, J. D., \& Schwartz, D. (2001). The dyadic nature of social information processing in boys' reactive and proactive aggression. Journal of Personality and Social Psychology, 80, 268-280.

Huesman, L. R., \& Guerra, N. G. (1997). Children's normative beliefs about aggression and aggressive behavior. Journal of Personality and Social Psychology, 72, 408-419.

Kristensen, C. H., Lima, J. S., Ferlin, M., Flores, R. Z., \& Hackmann, P. H. (2003). Fatores etiológicos da agressão física: uma revisão teórica. Estudos de Psicologia, 8, 175-184.

Liska, A. E., Richard, B. F., Chamlin, M., \& Baccaglini, W. (1984). Estimating attitude-behavior reciprocal effects within a theoretical specification. Social Psychology Quarterly, 47, 15-23.
Lochman E. J., \& Dodge K. A. (1994). Social cognitive processes of severely violent, moderately aggressive and nonagressive boys. Journal of Consulting and Clinical Psychology, 62, 366-374.

Mayeux, L., \& Cillessen, A. H. (2003). Development of social problem solving in early childhood: stability, change, and associations with social competence. Journal of Genetic Psychology, 164, 153-173.

Milich, R., \& Dodge, K. A. (1984). Social information processing in child psychiatric populations. Journal of Abnormal Child Psychology, 12, 471489.

Mize, J., \& Cox, R. (1990). Social knowledge and social competence: number and quality of strategies as predictors of peer behavior. The Journal of Genetic Psychology, 151, 117-127.

Pakaslahti, L. (2000). Children's and adolescents' aggressive behavior in context? The development and application of aggressive problem-solving strategies. Aggression and Violent Behavior, 5, 467-490.

Pakaslahti, L., \& Keltikangas-Jarvinen, L. (1998). Types of aggressive behavior among aggressive-preferred, aggressive non-preferred, non-aggressive preferred and non-aggressive non-preferred 14-year-old adolescents. Personality and Individual Differences, 24(6), 821-828.

Pettit, G. S., Dodge, K. A., \& Brown, M. M. (1988). Early family experience, social problem solving patterns, and children's social competence. Child Development, 59, 107-120.

Quiggle, N., Garber, J., Panak, W., \& Dodge, K. A. (1992). Social-information processing in aggressive and depressive children. Child Development, 63 , 1305-1320.

Richard, B. A., \& Dodge, K. A. (1982). Social maladjustment and problem solving in school-aged children. Journal of Consulting and Clinical Psychology, 50, 226-233.

Shahinfar, A., Kupersmidt, J. B., \& Matza, L. S. (2001). The relation between exposure to violence and social information processing among incarcerated adolescents. Journal of Abnormal Psychology, 110, 136-141.

Slaby, G. R., \& Guerra, N. G. (1988). Cognitive mediators of aggression in adolescents offenders. Assessment Development Psychology, 24, 580-588.

Smolensky, P. (1990). Tensor product variable binding and the representation of symbolic structures in connectionist network. Artificial Intelligence, 46, 159-216.

Steinberg, M. S., \& Dodge, K. A. (1982). Attributional bias in aggressive adolescents boys and girls. Journal of Social and Clinical Psychology, 1, 312-321.

Tur-Kaspa, H. (2004). Social-information-processing skills of kindergarten children with developmental learning disabilities. Learning Disabilities Research and Practice, 19, 3-11.

Silvio José Lemos Vasconcellos, mestre em Ciências Criminais pela Pontifícia Universidade Católica do Rio Grande do Sul, é professor de Psicologia Jurídica na FACCAT - Faculdades de Taquara (RS). Endereço para correspondência: Av. Oscar Martins Rangel, 4500 (RS 115); Taquara, RS; CEP 95600-000. Tel.: (51) 35416600. Fax: (51) 3541-6626. E-mail: silvv@pop.com.br

Patrícia Picon, doutora em Psiquiatria pela Universidade Federal do Rio Grande do Sul, é professora no Departamento de Psiquiatria e Medicina Legal da Faculdade de Medicina da Pontifícia Universidade Católica do Rio Grande do Sul.

Laura Pithan Prochnow, mestre em Psicologia do Desenvolvimento pela Universidade Federal do Rio Grande do Sul, é professora no Curso de Psicologia da Universidade Luterana do Brasil, em Santa Maria, RS.

Gabriel José Chittó Gauer, pós-doutor pela University of Maryland at College Park (EUA), é professor nos programas de pós-graduação em Psicologia e em Ciências Criminais, ambos da Pontifícia Universidade Católica do Rio Grande do Sul. 